\title{
From Colossal to Zero: Controlling the Anomalous Hall Effect in Magnetic Heusler Compounds via Berry Curvature Design
}

\author{
Kaustuv Manna, ${ }^{1, *}$ Lukas Muechler, ${ }^{1,2, *}$ Ting-Hui Kao, ${ }^{1,3}$ Rolf Stinshoff, ${ }^{1}$ Yang Zhang, ${ }^{1}$ Johannes Gooth, ${ }^{1}$ Nitesh Kumar, \\ Guido Kreiner, ${ }^{1}$ Klaus Koepernik, ${ }^{4}$ Roberto Car, ${ }^{2}$ Jürgen Kübler, ${ }^{1,5}$ Gerhard H. Fecher, ${ }^{1}$ Chandra Shekhar, ${ }^{1}$ \\ Yan Sun, ${ }^{1}$ and Claudia Felser ${ }^{1, \dagger}$ \\ ${ }^{1}$ Max Planck Institute for Chemical Physics of Solids, 01187 Dresden, Germany \\ ${ }^{2}$ Department of Chemistry, Princeton University, Princeton, New Jersey 08544, USA \\ ${ }^{3}$ Department of Physics, National Sun Yat-Sen University, Kaohsiung 804, Taiwan \\ ${ }^{4}$ IFW Dresden, P.O. Box 270116, 01171 Dresden, Germany \\ ${ }^{5}$ Institut für Festkörperphysik, Technische Universität Darmstadt, D-64289 Darmstadt, Germany
}

(Received 3 July 2018; revised manuscript received 25 September 2018; published 11 December 2018)

Since the discovery of the anomalous Hall effect (AHE), the anomalous Hall conductivity (AHC) has been thought to be zero when there is no net magnetization. However, the recently found relation between the intrinsic AHE and the Berry curvature predicts other possibilities, such as a large AHC in noncolinear antiferromagnets with no net magnetization but net Berry curvature. Vice versa, the AHE in principle could be tuned to zero, irrespective of a finite magnetization. Here, we experimentally investigate this possibility and demonstrate that the symmetry elements of Heusler magnets can be changed such that the Berry curvature and all the associated properties are switched while leaving the magnetization unaffected. This enables us to tune the AHC from $0 \Omega^{-1} \mathrm{~cm}^{-1}$ up to $1600 \Omega^{-1} \mathrm{~cm}^{-1}$ with an exceptionally high anomalous Hall angle up to $12 \%$, while keeping the magnetization the same. Our study shows that the AHC can be controlled by selectively changing the Berry curvature distribution, independent of the magnetization.

DOI: 10.1103/PhysRevX.8.041045

Subject Areas: Condensed Matter Physics, Magnetism

In conventional metals or semiconductors, a transverse voltage is generated due to the Lorentz force when a magnetic field is applied perpendicular to an applied electric current. This effect leads to an antisymmetric contribution to the off-diagonal electrical resistivity, which is generally known as the Hall effect. In ferro- or ferrimagnets it is believed that the spontaneous magnetization generates an additional transverse voltage due to the material's spin-orbit coupling (SOC), which causes carriers to be deflected by the magnetic moments of the host solid. This effect is known as the anomalous Hall effect (AHE) [1-3]. The AHE has two different contributions, an extrinsic contribution from scattering and an intrinsic contribution from the band structure $[1,4]$. For a long time it was believed that the anomalous Hall

\footnotetext{
* Corresponding author.

kaustuvmanna@gmail.com

Corresponding author.

felser@cpfs.mpg.de

*Present address: Center for Computational Quantum Physics, The Flatiron Institute, New York, New York 10010, USA.

Published by the American Physical Society under the terms of the Creative Commons Attribution 4.0 International license. Further distribution of this work must maintain attribution to the author(s) and the published article's title, journal citation, and DOI.
}

conductivity (AHC) scales with the sample's magnetization. According to this notion, any ferromagnetic material exhibits an AHE, but it is zero for an antiferromagnet, owing to the compensation of the magnetic sublattices $(M=0)$. Therefore, the AHE has been considered as the key signature of finite magnetization in ferromagnets or ferrimagnets. However, only recently, it was realized that the intrinsic contribution to the AHE is not directly related to the sample magnetization of a material but derives more generally from its net Berry curvature.

The Berry curvature distribution in materials is a property of the band structure that determines the topological aspects of a material [2,5-7]. A necessary condition for a finite net Berry curvature and thus a nonzero AHE is the absence of symmetries that reverse the sign of the local Berry curvature in the Brillouin zone (BZ) when reversing the sign of the momentum vector, e.g., time-reversal symmetry and mirror operations. In contrast to previous beliefs it is thus possible to control the Berry curvature and intrinsic AHE by suitable manipulations of symmetries and band structures, independent of the finite value of the magnetization [8]. As a result of these considerations, a strong AHC was predicted in the noncollinear antiferromagnetic systems like $\mathrm{Mn}_{3} \mathrm{Ir}$ [9], $\mathrm{Mn}_{3} \mathrm{Ge}$, and $\mathrm{Mn}_{3} \mathrm{Sn}$ [10], etc., and then experimentally observed in $\mathrm{Mn}_{3} \mathrm{Ge}$ [11] as well as $\mathrm{Mn}_{3} \mathrm{Sn}[12,13]$. Moreover, very recently, a large intrinsic AHE has been found in magnetic Weyl semimetals 
with broken time-reversal symmetry that depends on the separation of the Weyl nodes in momentum space [1,14-17]. In Weyl semimetals, the Weyl point acts as the monopole of Berry curvature, where a topological invariant, the Chern number, can be assigned to each Weyl node $[18,19]$. Therefore, one can tune the AHC via the symmetry and topological band structure without considering net magnetic moments [20].

In ferromagnetic materials, the distinction whether the magnetization or the Berry curvature is the origin of the AHE is more subtle: While most ferromagnets show a finite AHE that is proportional to the net magnetization of the sample, the AHE in principle could be tuned to zero, irrespective of the magnetization value. For a set of bands, the Berry curvature depends on how it is connected throughout the BZ [21]. In a magnetic semiconductor without band inversion, the AHC vanishes, as the occupied valence states can be adiabatically connected to the topologically trivial vacuum [Fig. 1(a)]. In a doped magnetic semiconductor or topologically trivial magnetic metal, the AHC can take small-to-large values depending on the details of the band structure; it usually follows the density of states (DOS) [Fig. 1(b)]. The energy dependence of the AHC is constrained if there is a trivial band gap close in energy, where the AHC has to vanish. In topological metals, such as Weyl semimetals or nodal line semimetals, the connectivity of the occupied bands is nontrivial owing to the band crossings. While the Berry curvature exhibits a strong peak close to the crossing points, the DOS vanishes, and the AHC peaks sharply in energy, with giant values [Fig. 1(c)]. The inverse relation between the DOS and AHC leads to a large anomalous Hall angle (AHA), unlike the small AHA in regular magnetic metals.

At a symmetry-protected crossing, e.g., in nodal line semimetals, the removal of the protecting symmetry leads to a gapping of the crossings, and the band structure

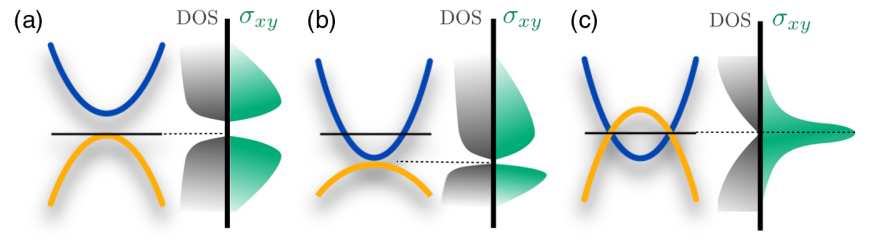

(d)

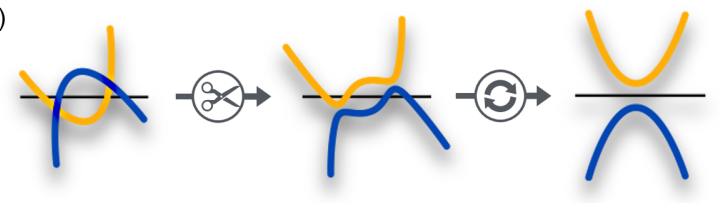

FIG. 1. Band structure, density of states, and AHC of (a) a trivial magnetic semiconductor, (b) a trivial metal, and (c) a topological metal. (d) Change in the topology of a topological metal by gapping out the crossing points via symmetry breaking or other strong perturbations. The gapped band structure is topologically equivalent to that of a trivial semiconductor. becomes topologically equivalent to that of a semiconductor [Fig. 1(d)]. We define a band structure as semiconducting in a topological sense, if it can be adiabatically deformed into the band structure of a gapped trivial semiconductor, even if it would be metallic from a transport perspective.

In this paper, we demonstrate these principles theoretically and experimentally and show that the AHC can be selectively tuned from 0 to $1600 \Omega^{-1} \mathrm{~cm}^{-1}$ in magnetic Heusler compounds via suitable manipulations of the symmetries and band structures of the materials. In contrast to previous studies, where a finite AHE has been observed without a net magnetization, we here show that the AHE can be tuned to zero despite a finite magnetization. Magnetic Heusler compounds are excellent materials for this purpose owing to the diverse possibilities for tuning the electronic and magnetic structure by varying the composition $[22,23]$. The large AHC and high Curie temperature $\left(T_{C}\right)$ allow us to obtain giant AHAs of up to $12 \%$ at the room temperature.

Ternary Heusler compounds with the formula $X_{2} Y Z$ (where $X$ and $Y$ are transition metals, and $Z$ is a main group element) crystallize in a face-centered cubic lattice with either space group (SG) $F m \overline{3} m$ (225) (the regular Heusler structure) or SG $F \overline{4} 3 m$ (216) (the inverse Heusler structure). In the regular Heusler structure, the $X$ atoms occupy the Wyckoff position $8 c(1 / 4,1 / 4,1 / 4)$, whereas the $Y$ and $Z$ atoms occupy the Wyckoff positions $4 b(1 / 2,1 / 2,1 / 2)$ and $4 a(0,0,0)$ with $\mathrm{Cu}_{2} \mathrm{MnAl}\left(L 2_{1}\right)$ as the prototype [24]. However, in the inverse Heusler structure, atoms in the $4 b$ position replace half of the $8 c$ atoms, and the revised atomic arrangement becomes $X[4 d(1 / 4,1 / 4,1 / 4)] X[4 b(1 / 2,1 / 2,1 / 2)]$ $Y[4 c(3 / 4,3 / 4,3 / 4)] Z[4 a(0,0,0)]$, with $\mathrm{Li}_{2} \mathrm{AgSb}$ as the prototype. The magnetic ground state (ferromagnetic, ferrimagnetic, or antiferromagnetic) of Heusler compounds is controlled by the interatomic distances of the corresponding $X$ and $Y$ atoms and follows the Slater-Pauling rules $[25,26]$. Further, $T_{C}$ generally scales with the sum of the local moments and can reach $1200 \mathrm{~K}$ [27].

We compare the structures of regular and inverse Heusler compounds in Figs. 2(a)-2(c). The $L 2_{1}$ structure (225) of regular Heusler compounds is symmorphic, and the crystallographic point group is $O_{h}$. Upon changing to the inverse Heusler structure, the structure loses inversion symmetry, and the point group reduces to $T_{d}$. Figures $2(\mathrm{~d})-$ 2(f) show the band structures along the high-symmetry points of three representative compounds. Evidently, the variation in the number of valance electrons $\left(N_{V}\right)$ affects only the position of the Fermi energy $E_{F}$ within the same symmetry group of compounds, as shown for $\mathrm{Co}_{2} \mathrm{MnGa}\left(N_{V}=28\right)$ and $\mathrm{Co}_{2} \mathrm{VGa}\left(N_{V}=26\right)$. In both compounds, the spin-up electrons form a topological nodal line, while the spin-down electrons possess a continuous gap throughout the BZ. The Fermi level lies in the band gap for $\mathrm{Co}_{2} \mathrm{VGa}$, while a small hole pocket is formed for 
(a)

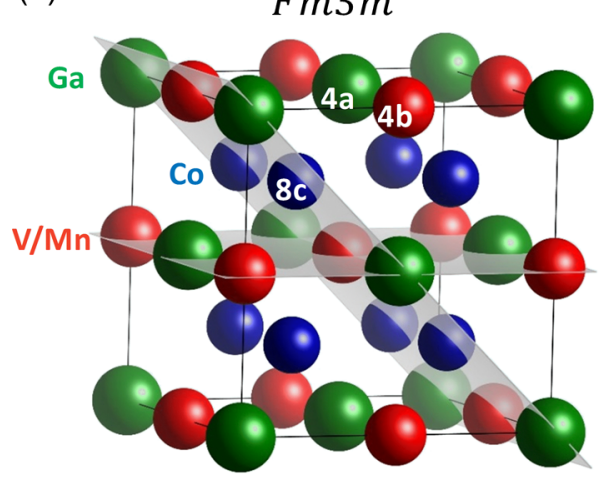

(d)
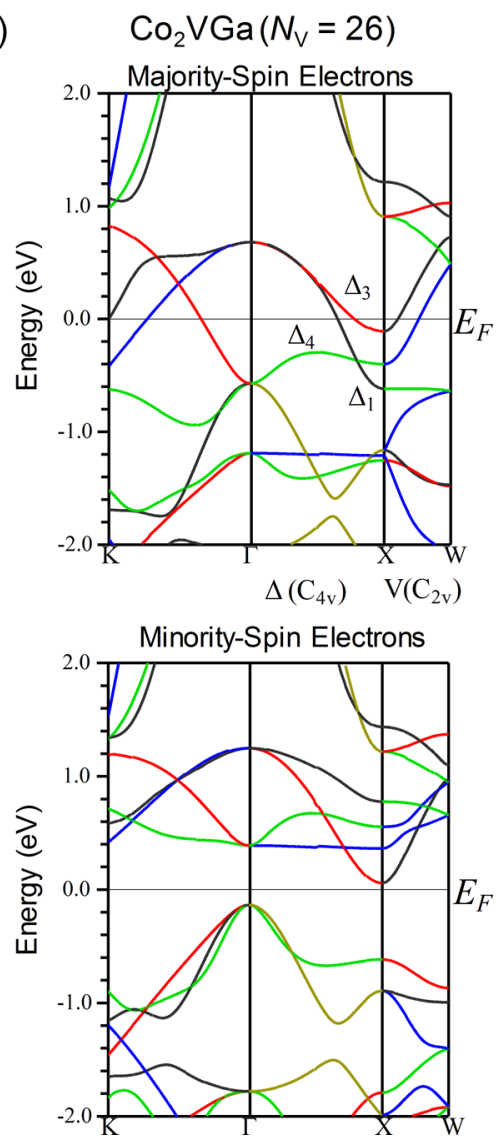

(b)

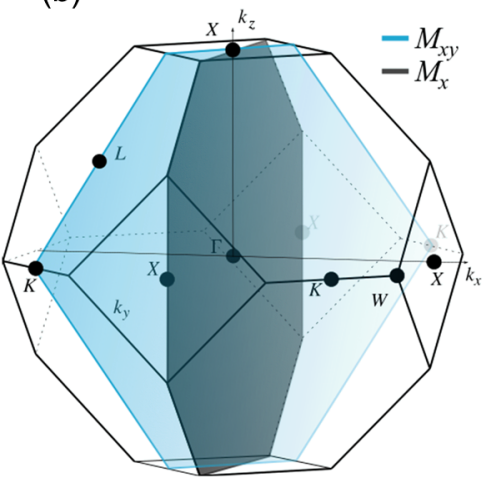

(e) $\quad \mathrm{Co}_{2} \mathrm{MnGa}\left(N_{\mathrm{V}}=28\right)$
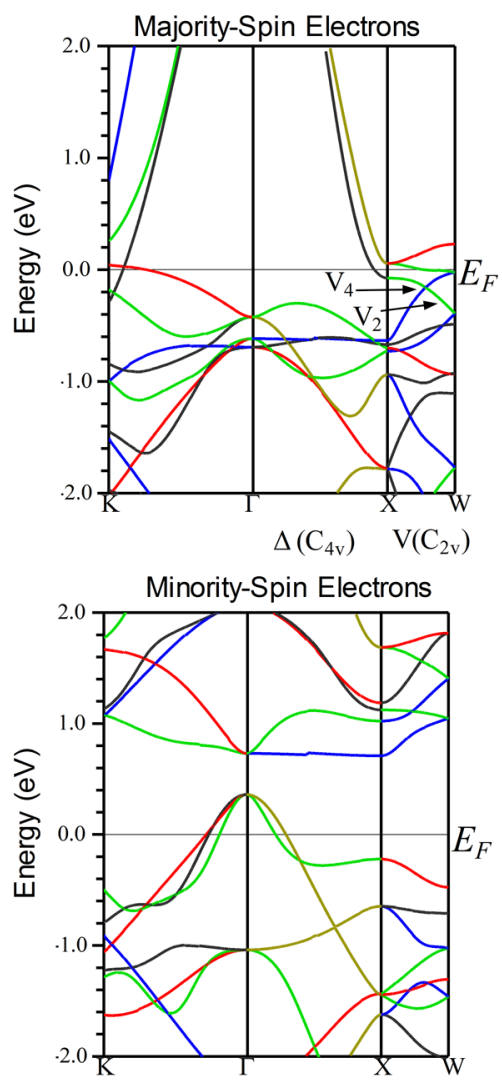

(c) $F \overline{4} 3 m$

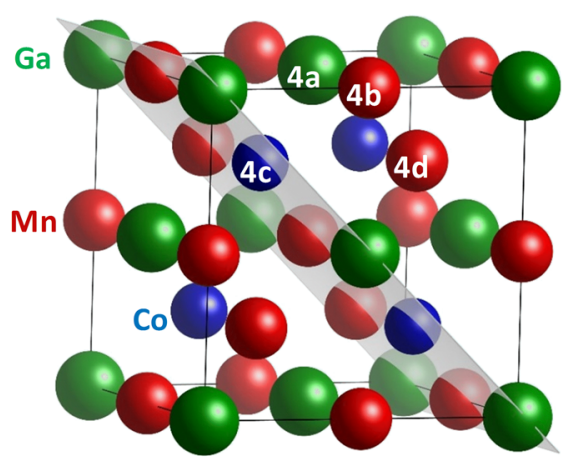

(f)
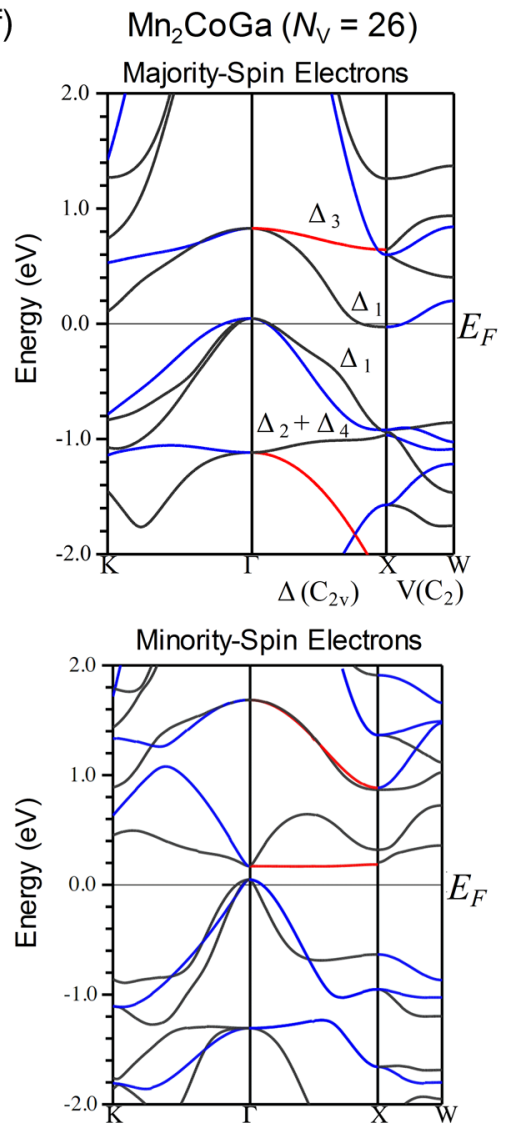

FIG. 2. (a) Lattice arrangements and (b) first bulk BZ for the regular Heusler compounds $\left(\mathrm{Co}_{2} \mathrm{VGa}\right.$ and $\left.\mathrm{Co}_{2} \mathrm{MnGa}\right)$ with $\mathrm{SG} F m \overline{3} m$. Corresponding high-symmetry points of the $\mathrm{BZ}$ are indicated, along with the allowed mirror symmetries. (c) Lattice arrangements for the inverse Heusler compounds $\left(\mathrm{Mn}_{2} \mathrm{CoGa}\right)$ with $\mathrm{SG} F \overline{4} 3 \mathrm{~m}$. The only allowed type of mirror plane is highlighted. Computed band structure of (d) $\mathrm{Co}_{2} \mathrm{VGa}$, (e) $\mathrm{Co}_{2} \mathrm{MnGa}$, and (f) $\mathrm{Mn}_{2} \mathrm{CoGa}$. Colors represent different irreducible representations (denoted by $\Delta$ and $\mathrm{V}$ ) of the little groups at each $k$.

$\mathrm{Co}_{2} \mathrm{MnGa}$. The band structure changes only slightly as the crystal symmetry changes from $225\left(\mathrm{Co}_{2} \mathrm{VGa}\right)$ to 216 $\left(\mathrm{Mn}_{2} \mathrm{CoGa}\right)$, whereas the total magnetic moment and $N_{V}$ remain the same. However, this symmetry change converts the topological nodal line semimetal $\mathrm{Co}_{2} \mathrm{VGa}$ into the trivial metal $\mathrm{Mn}_{2} \mathrm{CoGa}$ [Fig. 1(d)]. Being topologically nontrivial, $\mathrm{Co}_{2} \mathrm{VGa}$ and $\mathrm{Co}_{2} \mathrm{MnGa}$ should display an AHE with a peak close to the Fermi level [Fig. 1(c)]. In contrast, the AHE of $\mathrm{Mn}_{2} \mathrm{CoGa}$ should vanish at an energy close to the Fermi level, as it is topologically equivalent to a regular semiconductor [Fig. 1(a)].

Figures 3(a) and 3(c) show the $M-H$ hysteresis loops of single crystals of $\mathrm{Co}_{2} \mathrm{VGa}$ and $\mathrm{Mn}_{2} \mathrm{CoGa}$, respectively, at temperatures between 2 and $300 \mathrm{~K}$. The saturation magnetizations of the samples correspond to $M_{S}=2.0$ and $2.05 \mu_{B}$ at $2 \mathrm{~K}$, respectively, and decrease with increasing temperature. Our measured $M_{S}$ is in good agreement with the spin magnetic moment predicted by the Slater-Pauling 

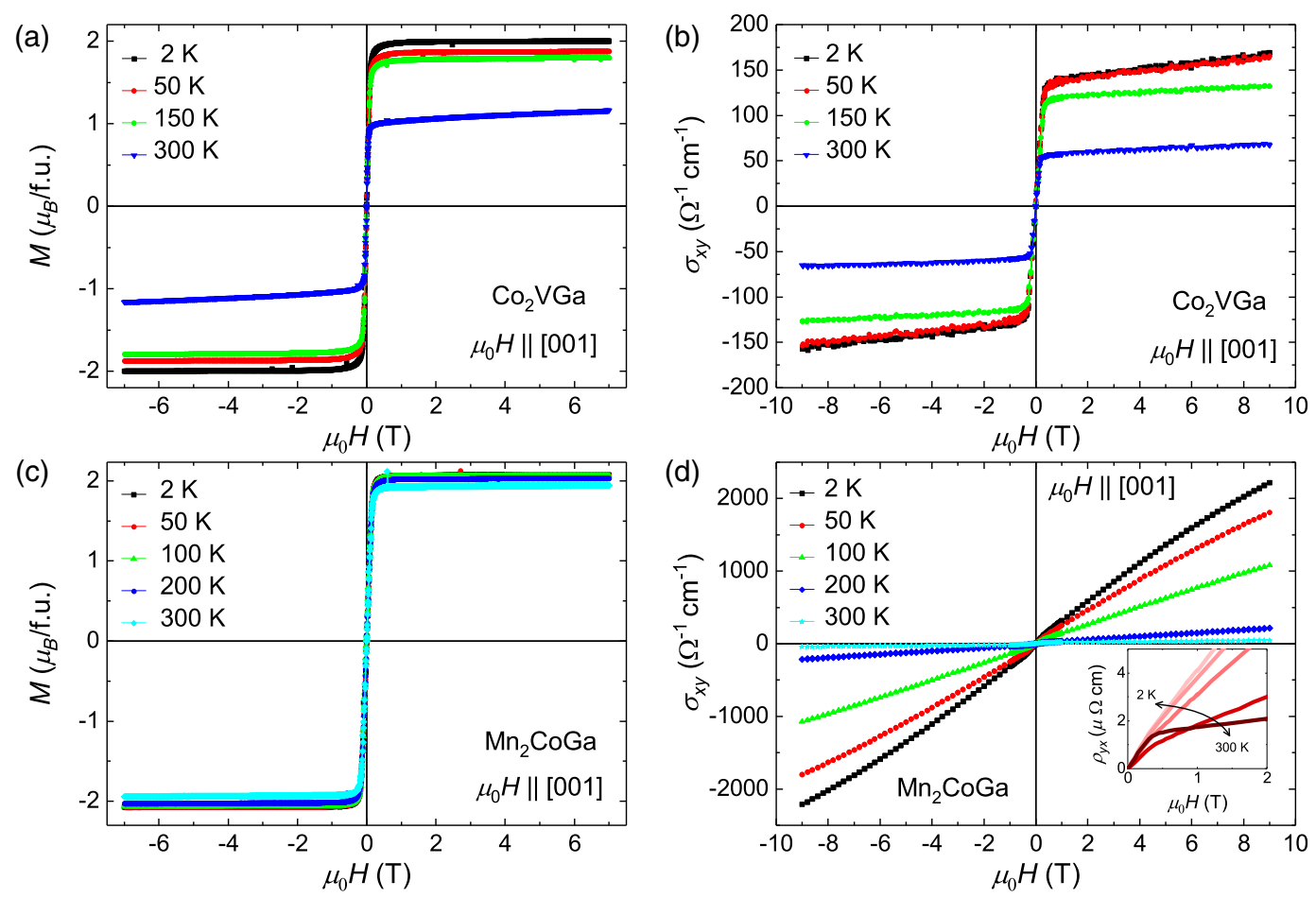

FIG. 3. (a),(c) Magnetic-field-dependent magnetization and (b),(d) corresponding field- and temperature-dependent AHC [ $\left.\sigma_{x y}\right]$ of (a),(b) $\mathrm{Co}_{2} \mathrm{VGa}$ and (c),(d) $\mathrm{Mn}_{2} \mathrm{CoGa}$ single crystals with field along the [001]. Inset of (d) shows a magnified view of the field-dependent Hall resistivity $\left[\rho_{y x}\right.$ ] of $\mathrm{Mn}_{2} \mathrm{CoGa}$ at various temperatures from 2 to $300 \mathrm{~K}$ in the low-field region. Here f.u. is formula unit.

rule, $M_{S}=\left(N_{V}-24\right) \mu_{B}[25,26]$. Temperature-dependent magnetization measurements reveal Curie temperatures of $328 \mathrm{~K}$ for $\mathrm{Co}_{2} \mathrm{VGa}$ and $715 \mathrm{~K}$ for $\mathrm{Mn}_{2} \mathrm{CoGa}$ [see Fig. S4 in the Supplemental Material (SM) [28] ].

Having established their similar saturation magnetization, we now discuss the AHE of both compounds. The total Hall resistivity $\rho_{y x}$ is expressed as

$$
\rho_{y x}=R_{0} \mu_{0} H+\rho_{y x}^{\mathrm{AHE}}
$$

where $R_{0}$ is the linear Hall coefficient and $\rho_{y x}^{\mathrm{AHE}}$ is the total anomalous Hall contribution. In the absence of any noncollinear spin textures, $\rho_{y x}^{\mathrm{AHE}}$ is commonly believed to scale with the spontaneous magnetization of materials $[1,4,29]$. However, this does not hold true for the topological magnetic materials. We calculate the Hall conductivity from the diagonal and off-diagonal components of the resistivity tensor as

$$
\sigma_{x y}=\rho_{y x} /\left(\rho_{x x}^{2}+\rho_{y x}^{2}\right)
$$

where $\rho_{x x}$ is the longitudinal resistivity. All known materials with ferromagnetic or ferrimagnetic ordering that exhibit spontaneous magnetization show an AHE [1]. $\mathrm{Co}_{2} \mathrm{VGa}$ obeys this rule very well, as seen in the summary of magnetotransport measurement in Fig. 3(b), with $B$ along the [001] direction. $\sigma_{x y}(B)$ increases linearly for a small applied magnetic field, and anomalous behavior is observed up to $\sim 0.5 \mathrm{~T}$. At the higher magnetic fields, the Hall resistivity slowly changes depending on $R_{0}$. However, at lower $B$, where the spins are not saturated, $\rho_{y x}$ is expected to have a contribution from the topological component. We estimate the AHC $\sigma^{A}$, to be $137 \Omega^{-1} \mathrm{~cm}^{-1}$ at $2 \mathrm{~K}$ by extrapolating the high field $\sigma_{x y}(B)$ data to the $B \rightarrow 0$ value. $\sigma^{A}$ gradually decreases at higher temperature to $58 \Omega^{-1} \mathrm{~cm}^{-1}$ at $300 \mathrm{~K}$. The Hall coefficient $R_{0}$ is calculated from the high field slope of $\rho_{y x}(B)$ as $4.6 \times 10^{-4} \mathrm{~cm}^{3} / \mathrm{C}$ at $2 \mathrm{~K}$. The sign of $R_{0}$ divulges important information about the type of charge carrier involved in transport. The majority charge carriers in $\mathrm{Co}_{2} \mathrm{VGa}$ are clearly the hole type in the entire measured temperature range (see Fig. S5 in the SM).

In contrast, $\mathrm{Mn}_{2} \mathrm{CoGa}$ shows very different anomalous Hall behavior. Figure 3(d) illustrates the Hall conductivity at various temperatures. Interestingly, $\sigma_{x y}$ increases linearly with the field, similar to a normal Hall effect. This is an exceptional observation in a metallic magnetic material with a large magnetic moment of $2 \mu_{B}$ and differs markedly with other similar compounds reported in the literature [1,30]. The calculated $R_{0}$ is $0.035 \mathrm{~cm}^{3} / \mathrm{C}$ at $2 \mathrm{~K}$ for $\mathrm{Mn}_{2} \mathrm{CoGa}$ and decreases sharply (by a factor of 10 ) to $0.0035 \mathrm{~cm}^{3} / \mathrm{C}$ at $300 \mathrm{~K}$. Consequently, $\sigma_{x y}(B)$ decreases remarkably to a negligible value at $300 \mathrm{~K}$ compared to that at $2 \mathrm{~K}$ [Fig. 3(d)]. The charge carriers are of the hole type. For all the above measurements, the magnetic field $B$ and current were applied along the [001] and [100] directions, 
respectively. However, $B$ along [011] or [1111] configuration shows similar properties, indicating small anisotropy in the sample.

We now want to understand the unusual behavior of $\mathrm{Mn}_{2} \mathrm{CoGa}$ theoretically. As discussed earlier, the inverse Heusler $\mathrm{Mn}_{2} \mathrm{CoGa}$ can be considered as the symmetryreduced counterpart of regular Heusler $\mathrm{Co}_{2} \mathrm{VGa}$, which has the same $N_{V} \cdot \mathrm{Co}_{2} \mathrm{VGa}$ belongs to the space group of $F m \overline{3} m$, and the topological properties in $\mathrm{Co}_{2} \mathrm{VGa}$ are due to the three mirror planes $M_{x}, M_{y}$, and $M_{z}$. Without considering spin-orbit coupling, the full Hamiltonian can be decomposed into the direct product of a spin-up Hamiltonian and spin-down Hamiltonian, i.e., the band degeneracy of each spin channel is decided by the corresponding Hamiltonian. Owing to the mirror symmetry, the band inversion between the bands with opposite mirror eigenvalue forms three gapless nodal lines in the $k_{x}=0$, $k_{y}=0$, and $k_{z}=0$ mirror planes, respectively. Compared to $\mathrm{Co}_{2} \mathrm{VGa}$, the crucial difference in $\mathrm{Mn}_{2} \mathrm{CoGa}$ is the absent of these three mirror planes. Though they have the same $N_{V}$, the mirror-plane-protected gapless nodal lines do not exist in $\mathrm{Mn}_{2} \mathrm{CoGa}$ due to the lack of the three mirror planes.

After taking SOC into consideration, the symmetry of the system is reduced depending on the direction of magnetization, which leads to band anticrossings of the nodal lines and generates Weyl nodes near $E_{F}$ for regular Heusler (like $\mathrm{Co}_{2} \mathrm{VGa}, \mathrm{Co}_{2} \mathrm{MnGa}$, etc.) [21,31]. This is due to the fact that the mirror planes perpendicular to the magnetization do not preserve the direction of the spins, while the ones parallel do. For example, if we consider a magnetization along the $\hat{z}$ direction (as used in the experiment), the mirrors $M_{x}, M_{y}$ are no longer allowed symmetry operations, whereas $M_{z}$ remains a symmetry operation. Thus, an anticrossing band gap will only appear from the two nodal lines in the $k_{x}=0, k_{y}=0$ planes. However, some linear crossing points are still allowed due to the combined time-reversal and rotational symmetries, which are just the Weyl points [21,31,32]. In comparison with regular Heusler compounds such as $\mathrm{Co}_{2} \mathrm{VGa}$, the absence of nodal lines in inverse Heusler compounds (like $\mathrm{Mn}_{2} \mathrm{CoGa}$ ) without spin-orbit interaction generally leads to the absence of Weyl points.

Figures 4(a) and 4(b) show the computed spin-resolved DOS for $\mathrm{Co}_{2} \mathrm{VGa}$ and $\mathrm{Mn}_{2} \mathrm{CoGa}$, respectively. $\mathrm{Co}_{2} \mathrm{VGa}$ is clearly a half-metallic ferromagnet, i.e., one spin channel is gapped while the other one is metallic. In contrast, $\mathrm{Mn}_{2} \mathrm{CoGa}$ possesses one semimetallic spin channel with both electron and hole pockets. The other spin channel has a global band gap in the range of $\sim E_{F}+0.02$ to $\sim E_{F}+$ $0.1 \mathrm{eV}$ due to the absence of the mirror symmetries. The closely related compound $\mathrm{Mn}_{2} \mathrm{CoAl}$ is a spin-gapless semiconductor, in which one spin channel is gapped, whereas the other spin channel is semimetallic with a vanishing DOS at the Fermi energy [33].

We now discuss these band-structure effects on the AHC of both compounds (for details of the calculation, see the
Methods section). In the limit of weak SOC, the AHC is given as the sum of the conductivities for each spin species, $\sigma^{A}(\mu)=\sigma^{\uparrow A}(\mu)+\sigma^{\downarrow A}(\mu)$, where $\mu$ is the chemical potential [34]. For the half-metallic ferromagnet $\mathrm{Co}_{2} \mathrm{VGa}$, the states around the Fermi level arise only from the majority states, whereas the minority states exhibit a band gap of about $0.2 \mathrm{eV}$. Thus, the contribution to the AHC of the minority carriers remains constant throughout the band-gap energy window [Fig. 4(c)]. Because most of the slightly gapped nodal lines from SOC that generate a large Berry curvature, lie far from the Fermi level [Fig. 2(d)], the absolute value of the integrated Berry curvature of the spinup channel is not very large. We calculate an intrinsic AHC of $\sigma^{A}(\mu)=140 \Omega^{-1} \mathrm{~cm}^{-1}$ for $\mathrm{Co}_{2} \mathrm{VGa}$, which is consistent with the experiment.

In $\mathrm{Mn}_{2} \mathrm{CoGa}$, however, both spin species possess a finite AHC around the Fermi level [Fig. 4(d)]. The sign of the $\mathrm{AHC}$ of the spin-up electrons is opposite to that of the AHC of the spin-down electrons around $E_{F}$. Close to $E_{F}$, we can approximate $\sigma^{A}(\mu) \approx\left|\sigma^{\uparrow A}(\mu)\right|-\left|\sigma^{\downarrow A}(\mu)\right|$. Therefore, $\sigma^{A}$ can switch sign depending on which spin channel contributes the most to the overall AHC. At the charge neutrality point, the Berry curvature contribution of the spin-up channel is approximately $74 \Omega^{-1} \mathrm{~cm}^{-1}$ larger than that of the spin-down channel, resulting in a finite AHC. In our ab initio calculation, the compensation point $\sigma^{A}(\mu)=0$ lies about $0.1 \mathrm{eV}$ above the Fermi level, which corresponds to approximately 0.1 extra electrons per formula unit. As shown in the SM, our compound is slightly electron doped, which could explain the discrepancy between theory and experiment. More importantly, the presence of such a compensation point close to $E_{F}$ is a general topological property of compounds with an electronic structure topologically equivalent to that of a semiconductor, such as spin-gapless semiconductors. These occur in the inverse Heusler structure owing to breaking of the mirror symmetries if the corresponding full-Heusler compound with the same $N_{V}$ is a half-metallic nodal line semimetal.

Since the AHC can be obtained by the integral of the Berry curvature in the Brillouin zone, we can use the Berry curvature distribution in $k$ space of the two groups of Heusler compounds to correlate the band structure and corresponding AHC. As discussed, when $\mathrm{Co}_{2} \mathrm{VGa}$ is magnetized along the $z$ direction, the mirrors $M_{x}$ and $M_{y}$ are no longer symmetry planes, and a gapless nodal line exists only in the $k_{z}=0$ plane. The band structure of a gapless nodal line contains nonzero Berry curvature around it; however, it is helically distributed in the mirror plane, and the total flux through the mirror plane is zero. Thus, the gapless nodal line in the $k_{z}=0$ plane does not contribute to the intrinsic $\mathrm{AHC}$ of $\mathrm{Co}_{2} \mathrm{VGa}$. However, the nodal lines in the $k_{x}=0$ and $k_{y}=0$ planes are gapped by SOC, leading to band anticrossings, which forces the Berry curvature to be oriented along the magnetization direction. For example, in the $k_{y}=0$ plane, the $k$ points around the broken nodal 

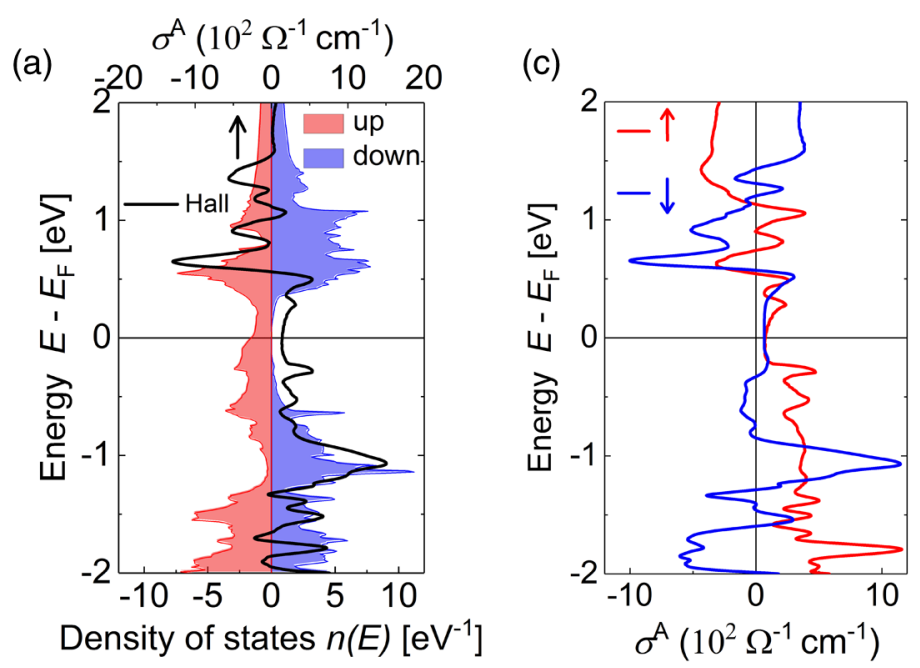

(e)
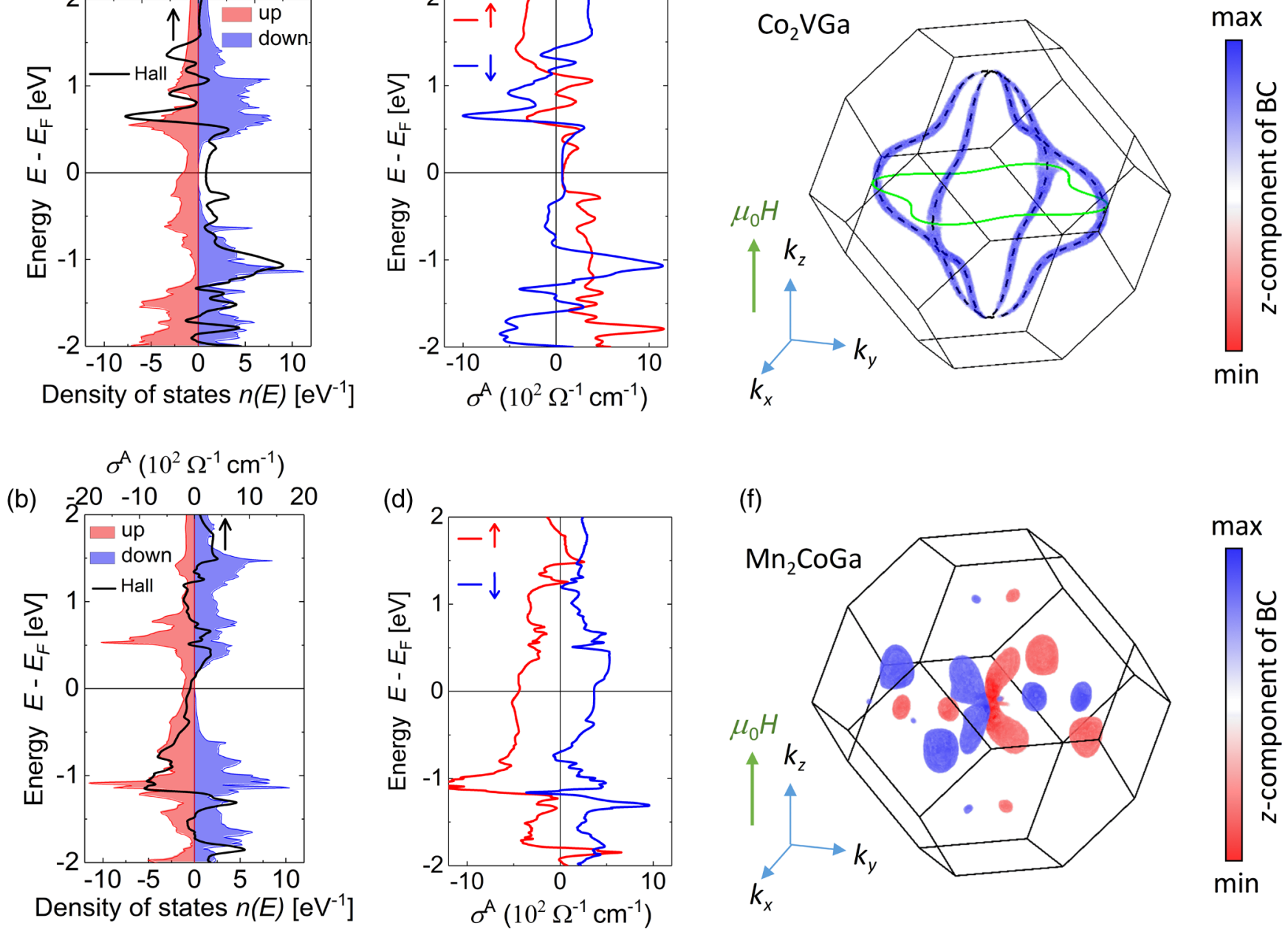

FIG. 4. Computed spin-resolved DOS for (a) $\mathrm{Co}_{2} \mathrm{VGa}$ and (b) $\mathrm{Mn}_{2} \mathrm{CoGa}$. Black lines represent the calculated net anomalous Hall conductivity $\left(\sigma^{A}\right)$ values. Red and blue represent the DOS for the majority and minority carriers, respectively. Hall contributions in the majority and minority spin channels for (c) $\mathrm{Co}_{2} \mathrm{VGa}$ and (d) $\mathrm{Mn}_{2} \mathrm{CoGa}$. Berry curvature distribution in the $\mathrm{BZ}$ for (e) $\mathrm{Co}_{2} \mathrm{VGa}$ and (f) $\mathrm{Mn}_{2} \mathrm{CoGa}$ calculated for all valence bands. The solid green line in (e) represents the protected nodal line due to the $M_{z}$ symmetry operation with magnetic field along $z$ direction. The dotted black lines are the gapped nodal lines which the Berry curvature (BC) distribution in $\mathrm{Co}_{2} \mathrm{VGa}$ follows.

lines are dominated by the negative $\Omega_{x y}$ components of the Berry curvature. The Berry curvature distribution in the Brillouin zone for $\mathrm{Co}_{2} \mathrm{VGa}$ is shown in Fig. 4(e). Because the nodal lines in the mirror planes have strong energy dispersion [31,35], only some parts of the nodal lines contribute to the AHC at the fixed Fermi level, resulting in an $\mathrm{AHC}$ of $\sim 140 \Omega^{-1} \mathrm{~cm}^{-1}$ at $E_{F}$.

The Berry curvature distribution in $\mathrm{Mn}_{2} \mathrm{CoGa}$ is very different due to the absence of a nodal line band structure [Fig. 4(f)]. In $\mathrm{Mn}_{2} \mathrm{CoGa}$ it contains both positive and negative hot spots, throughout the Brillouin zone. Therefore, during integration of the Berry curvature, hot spots of opposite sign cancel, producing a small AHC value. By doping or temperature effects, one can tune the chemical potential to the topologically required critical point where the positive and negative Berry curvatures are totally canceled out, yielding zero AHC. Interestingly, this is validated by our experiment, as shown in the inset of Fig. 3(d) for $\mathrm{Mn}_{2} \mathrm{CoGa}$. As the temperature increases up to $300 \mathrm{~K}$, a nonzero AHE is observed, and $\rho_{y x}(B)$ clearly scales with the sample's magnetization.

The obtained results show that the interplay of the crystal symmetry with the topological and geometrical properties of the Berry curvature provides a powerful framework to control the AHC, independent of the magnetization $[17,34]$. We condense our findings in Fig. 5, which presents a strategy for tuning the electronic and topological properties of Heusler compounds. In regular Heusler compounds, we find a half-metallic nodal line protected by mirror symmetry at different valence electron counts. By suitable 
(a)

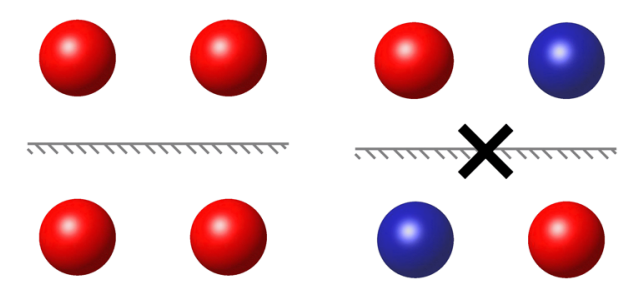

(c)

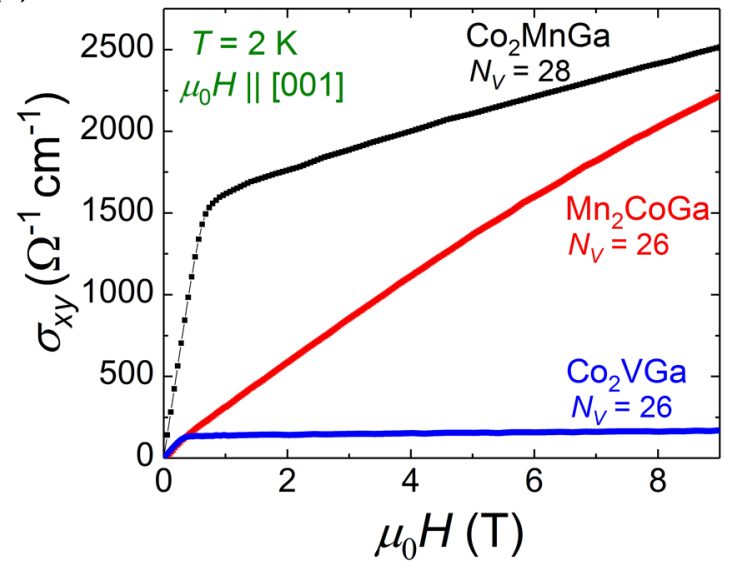

(b) $N_{\mathrm{V}}\left[\mathrm{M}_{\mathrm{S}}, \mu_{\mathrm{B}} /\right.$ f.u. $]$

$F m \overline{3} m(F \overline{4} 3 m)$

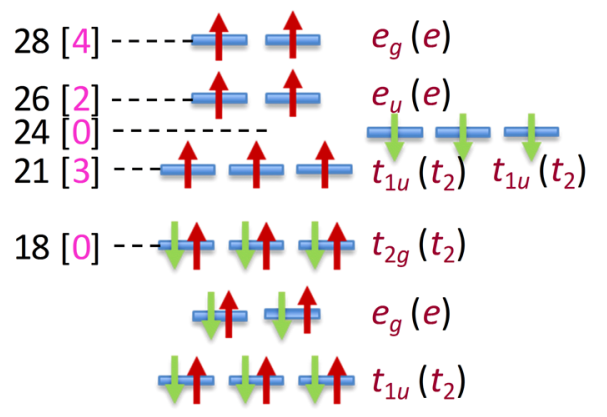

(d)

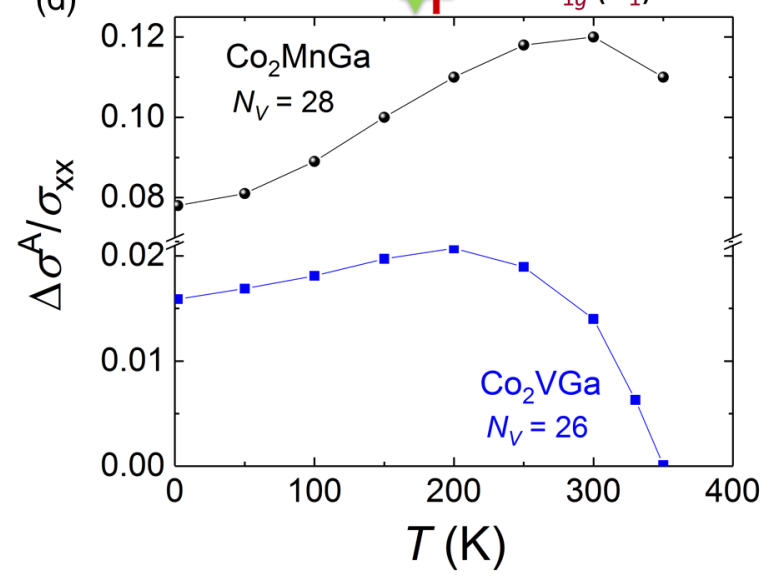

(g)

CoFeMnSi $\left(N_{V}=28\right)$

(h) $\mathrm{Co}_{2} \mathrm{MnGa}\left(N_{\mathrm{V}}=28\right)$

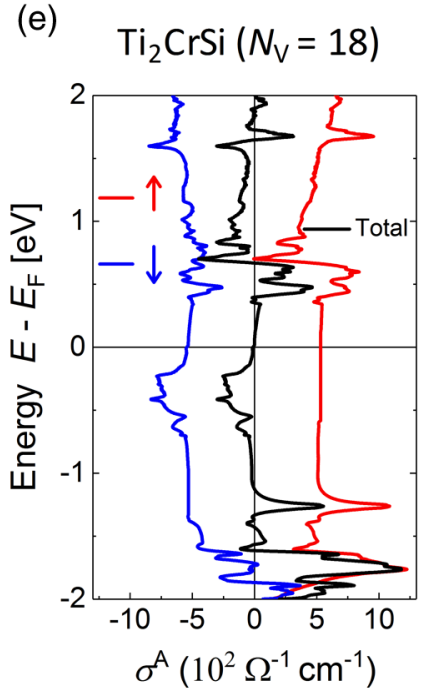

(f) $\operatorname{CoVTiAl}\left(N_{\mathrm{V}}=21\right)$

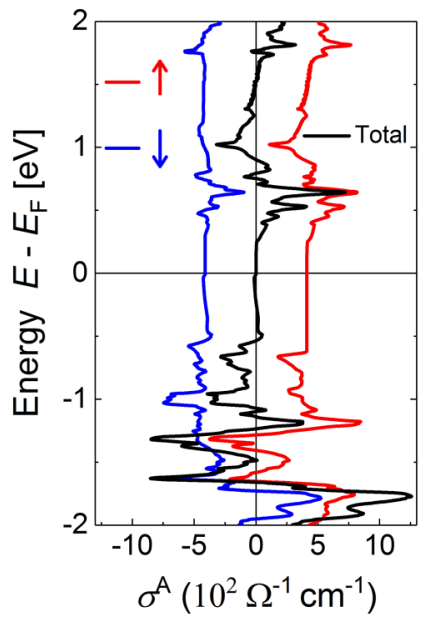

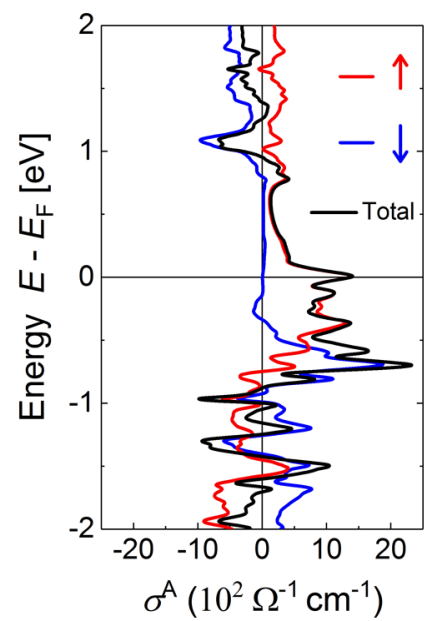

FIG. 5. (a) Breaking of mirror symmetry by chemical substitution in the compound. (b) Schematic representation of the distribution of valence electrons in various electronic energy levels for both spin channels and the corresponding net magnetic moment. (c) Experimentally observed Hall conductivity of three magnetic Heusler compounds with various $N_{V}$. (d) Temperature dependence of the anomalous Hall angle for two regular Heusler compounds, $\mathrm{Co}_{2} \mathrm{MnGa}$ and $\mathrm{Co}_{2} \mathrm{VGa}$. Hall contributions of the majority and minority spin channels and the net anomalous Hall conductivity for (e) $\mathrm{Ti}_{2} \mathrm{CrSi}$, (f) CoVTiAl, (g) $\mathrm{CoFeMnSi}$, and (h) $\mathrm{Co}_{2} \mathrm{MnGa}$.

chemical substitution, one may destroy the mirror symmetry of the compound [Fig. 5(a)]. The removal of certain mirror symmetries changes the electronic structure of a half-metallic nodal line semimetal into a trivial band structure very close to that of a spin-gapless semiconductor by removing the nodal lines. These changes induce corresponding changes in the Berry curvature and thus lead to a small or vanishing AHC. Because of the excellent tunability, one can easily manipulate the chemical potential in Heusler compounds by changing $N_{V}$ [Fig. 5(b)]. 
For example, $\sigma^{A}$ changes from $\sim 140 \Omega^{-1} \mathrm{~cm}^{-1}$ for $\mathrm{Co}_{2} \mathrm{VGa}\left(N_{V}=26\right)$ to $\sim 1600 \Omega^{-1} \mathrm{~cm}^{-1}$ for $\mathrm{Co}_{2} \mathrm{MnGa}$ $\left(N_{V}=28\right)$, which also possesses nodal lines around the Fermi level, although the compounds have different $N_{V}$.

Again, keeping the same $N_{V}$, when the crystal symmetry is altered from $O_{h}$ in $\mathrm{Co}_{2} \mathrm{VGa}$ to $T_{d}$ in $\mathrm{Mn}_{2} \mathrm{CoGa}, \sigma^{A}$ decreases to zero [Fig. 5(c)]. The maximum AHA, $\Theta_{\mathrm{AHE}}=\Delta \sigma^{A} / \sigma_{x x}$, reaches a giant value of $\sim 12 \%$ for $\mathrm{Co}_{2} \mathrm{MnGa}$ at room temperature, at which the nodal line is closest to $E_{F}$ [Fig. 5(d)]. We conjecture that the nodal line dispersion and charge carrier concentration [Figs. 2(d) and 2(e)] control the value of $\Theta_{\mathrm{AHE}}$, which is why $\Theta_{\mathrm{AHE}}$ for $\mathrm{Co}_{2} \mathrm{VGa}$ is only about $2 \%$ [Fig. 5(d)].

Owing to the extensive tunability of Heusler compounds and the topological nature of our arguments, the compensated AHC should not be unique to $\mathrm{Mn}_{2} \mathrm{CoGa}$. Since all the spin-gapless Heusler semiconductors have similar Berry curvature distributions, the same principle generally applies among all of them, for example, $\mathrm{Ti}_{2} \mathrm{CrSi}, \mathrm{CoVScSi}$, and $\mathrm{CoFeMnSi}$. In the $\mathrm{SM}$, we list a series of spin-gapless semiconductor candidate compounds, classified according to the corresponding $N_{V}$ that should show a compensated AHC near $E_{F}$. Therefore, we can add a new rule to the simple rules of Heusler compounds [24]: For each spingapless semiconductor, there exists a corresponding halfmetallic full-Heusler compound with a nodal line in the majority spin channel and Weyl nodes close to $E_{F}$ with the same $N_{V}$, and vice versa. This implies that there should be many more compounds with a large AHC and AHA in the Heusler family. The AHC of the full-Heusler compound will generally be large, whereas that of the inverse Heusler compound will be close to zero, as we illustrate for $\mathrm{CoFeMnSi}$ and $\mathrm{Co}_{2} \mathrm{MnGa}$, which have $N_{V}=28$ [Figs. 5(g) and 5(h), respectively].

Vice versa to previous studies, where a finite AHE has been observed without a net magnetization, we have showed that the AHE can be tuned to zero despite a finite magnetization. Thus, our results complement the recent investigations on the Berry curvature origin of the AHE. Our findings are general and can be extended to other classes of materials with finite magnetization, as one can control the topology by changing the magnetic space group. Thus, our work is relevant to the recent interest in topological classification of magnetic materials, as the topology often constrains the Berry curvature distribution [6]. By symmetry engineering, a metallic magnet can be converted to a topologically trivial semiconductor with zero AHC. The possibility to tune the AHE from zero to a colossal value, independent of the magnetization of the material, may be interesting for next-generation topospintronics applications. Additionally, topological semimetals with a high Curie temperature and large AHA, such as Heusler compounds, are excellent candidate materials for a confinement-induced quantum AHE in thin films.

Methods.-Single crystals of $\mathrm{Mn}_{2} \mathrm{CoGa}, \mathrm{Co}_{2} \mathrm{VGa}$, and $\mathrm{Co}_{2} \mathrm{MnGa}$ were grown using the Bridgman-Stockbarger crystal growth technique. First, stoichiometric amounts of high-purity premelted metals were in an alumina crucible using induction melting. Then the crushed powder was packed in a custom-designed sharp-edged alumina tube, which was sealed in a tantalum tube. Before crystal growth, the compound's melting point was determined using differential scanning calorimetry measurement. The samples were heated (to $1150^{\circ} \mathrm{C}$ for $\mathrm{Mn}_{2} \mathrm{CoGa}, 1450^{\circ} \mathrm{C}$ for $\mathrm{Co}_{2} \mathrm{VGa}$, and $1250^{\circ} \mathrm{C}$ for $\mathrm{Co}_{2} \mathrm{MnGa}$ ), held there for $10 \mathrm{~h}$ to ensure homogeneous mixing of the melt, and then slowly cooled to $750^{\circ} \mathrm{C}$. Single crystallinity was checked by white-beam backscattering Laue $\mathrm{x}$-ray diffraction at room temperature. The crystal structures were analyzed with a Bruker D8 VENTURE x-ray diffractometer using Mo-K radiation. Magnetization measurements were performed using a Quantum Design vibrating sample magnetometer (MPMS). The transport properties were characterized by a Quantum Design physical property measurement system (PPMS, ACT option).

The electronic band structure was calculated using density functional theory with the localized atomic orbital basis and the full potential as implemented in the fullpotential local-orbital (FPLO) code [36]. The exchange and correlation energies were considered in the generalized gradient approximation following the Perdew-BurkeErnzerhof parametrization scheme [37]. To calculate the $\mathrm{AHC}$, we project the Bloch wave functions into highsymmetry atomiclike Wannier functions, and construct the tight-binding model Hamiltonian. On the basis of the tightbinding model Hamiltonian, we calculated the AHC using the Kubo formula and clean limit [14]:

$$
\begin{aligned}
& \sigma_{x y}^{z}\left(E_{F}\right)=\frac{e^{2}}{\hbar}\left(\frac{1}{2 \pi}\right)^{3} \int_{\vec{k}} d \vec{k} \sum_{E(n, \vec{k})<E_{F}} f(n, \vec{k}) \Omega_{n, x y}^{z}(\vec{k}), \\
& \Omega_{n, x y}^{z}(\vec{k})=\operatorname{Im} \sum_{n^{\prime} \neq n} \frac{\left\langle u(n, \vec{k})\left|\hat{v}_{x}\right| u\left(n^{\prime}, \vec{k}\right)\right\rangle\left\langle u\left(n^{\prime}, \vec{k}\right)\left|\hat{v}_{y}\right| u(n, \vec{k})\right\rangle-(x \leftrightarrow y)}{\left[E(n, \vec{k})-E\left(n^{\prime}, \vec{k}\right)\right]^{2}},
\end{aligned}
$$

where $f(n, \vec{k})$ is the Fermi-Dirac distribution, $E(n, \vec{k})$ is the eigenvalue for the $n$th eigenstates of $|u(n, \vec{k})\rangle$ at the $\vec{k}$ point, and $\hat{v}_{x(y)}$ is the velocity operator. A $201 \times 201 \times 201 k$ grid was used in the integral. We also classify the Berry curvature $\Omega_{n, x y}^{z}(\vec{k})$ into two species, $\Omega_{n, x y}^{z, \uparrow}(\vec{k})$ and $\Omega_{n, x y}^{z, \downarrow}(\vec{k})$, using the expectation value $\hat{s}_{n, z}(\vec{k})$ of the wave 
function for the given band and $k$ point. The spin-resolved Berry phases $\sigma_{x y}^{z, \uparrow}\left(E_{F}\right)$ and $\sigma_{x y}^{z, \downarrow}\left(E_{F}\right)$ are obtained by taking the integral of $\Omega_{n, x y}^{z, \uparrow}(\vec{k})$ and $\Omega_{n, x y}^{z, \downarrow}(\vec{k})$, respectively, in the entire BZ.

This work was financially supported by the ERC Advanced Grant No. 291472 "Idea Heusler," ERC Advanced Grant No. 742068-TOPMAT, and Deutsche Forschungsgemeinschaft DFG under SFB 1143. T. H. K. acknowledges financial support from the Ministry of Science and Technology, Taiwan, under Grants No. 1052917-I-110-004 and No. 103-2112-M-110-010-MY3. L.M. is grateful for the hospitality of the MPI CPFS, where part of the work was conducted. We thank J. Noky for helping the Berry curvature calculations.

The authors declare no competing financial interests.

[1] N. Nagaosa, J. Sinova, S. Onoda, A. H. MacDonald, and N. P. Ong, Anomalous Hall Effect, Rev. Mod. Phys. 82, 1539 (2010).

[2] R. Karplus and J. M. Luttinger, Hall Effect in Ferromagnetics, Phys. Rev. 95, 1154 (1954).

[3] S. Onoda, N. Sugimoto, and N. Nagaosa, Quantum Transport Theory of Anomalous Electric, Thermoelectric, and Thermal Hall Effects in Ferromagnets, Phys. Rev. B 77, 165103 (2008).

[4] S. Onoda, N. Sugimoto, and N. Nagaosa, Intrinsic Versus Extrinsic Anomalous Hall Effect in Ferromagnets, Phys. Rev. Lett. 97, 126602 (2006).

[5] B. Bradlyn, L. Elcoro, J. Cano, M. G. Vergniory, Z. Wang, C. Felser, M. I. Aroyo, and B. A. Bernevig, Topological Quantum Chemistry, Nature (London) 547, 298 (2017).

[6] H. Watanabe, H. C. Po, and A. Vishwanath, Structure and Topology of Band Structures in the 1651 Magnetic Space Groups, Sci. Adv. 4, eaat8685 (2018).

[7] H. C. Po, A. Vishwanath, and H. Watanabe, SymmetryBased Indicators of Band Topology in the 230 Space Groups, Nat. Commun. 8, 50 (2017).

[8] R. Shindou and N. Nagaosa, Orbital Ferromagnetism and Anomalous Hall Effect in Antiferromagnets on the Distorted fcc Lattice, Phys. Rev. Lett. 87, 116801 (2001).

[9] H. Chen, Q. Niu, and A. H. MacDonald, Anomalous Hall Effect Arising from Noncollinear Antiferromagnetism, Phys. Rev. Lett. 112, 017205 (2014).

[10] J. Kübler and C. Felser, Non-Collinear Antiferromagnets and the Anomalous Hall Effect, Europhys. Lett. 108, 67001 (2014).

[11] A. K. Nayak et al., Large Anomalous Hall Effect Driven by a Nonvanishing Berry Curvature in the Noncolinear Antiferromagnet $\mathrm{Mn}_{3} \mathrm{Ge}$, Sci. Adv. 2, e1501870 (2016).

[12] S. Nakatsuji, N. Kiyohara, and T. Higo, Large Anomalous Hall Effect in a Non-Collinear Antiferromagnet at Room Temperature, Nature (London) 527, 212 (2015).

[13] N. H. Sung, F. Ronning, J. D. Thompson, and E. D. Bauer, Magnetic Phase Dependence of the Anomalous Hall Effect in $\mathrm{Mn}_{3} \mathrm{Sn}$ Single Crystals, Appl. Phys. Lett. 112, 132406 (2018).
[14] D. Xiao, M.-C. Chang, and Q. Niu, Berry Phase Effects on Electronic Properties, Rev. Mod. Phys. 82, 1959 (2010).

[15] A. A. Burkov, Anomalous Hall Effect in Weyl Metals, Phys. Rev. Lett. 113, 187202 (2014).

[16] J. Kübler and C. Felser, Weyl Points in the Ferromagnetic Heusler Compound $\mathrm{Co}_{2} \mathrm{MnAl}$, Europhys. Lett. 114, 47005 (2016).

[17] J. Kübler and C. Felser, Berry Curvature and the Anomalous Hall Effect in Heusler Compounds, Phys. Rev. B 85, 012405 (2012).

[18] B. Bradlyn, J. Cano, Z. Wang, M. G. Vergniory, C. Felser, R. J. Cava, and B. A. Bernevig, Beyond Dirac and Weyl Fermions: Unconventional Quasiparticles in Conventional Crystals, Science 353, aaf5037 (2016).

[19] N. P. Armitage, E. J. Mele, and A. Vishwanath, Weyl and Dirac Semimetals in Three Dimensional Solids, Rev. Mod. Phys. 90, 015001 (2018).

[20] K. Manna, Y. Sun, L. Muechler, J. Kübler, and C. Felser, Heusler, Weyl and Berry, Nat. Rev. Mater. 3, 244 (2018).

[21] Z. Wang, M. G. Vergniory, S. Kushwaha, M. Hirschberger, E. V. Chulkov, A. Ernst, N. P. Ong, R. J. Cava, and B. A. Bernevig, Time-Reversal-Breaking Weyl Fermions in Magnetic Heusler Alloys, Phys. Rev. Lett. 117, 236401 (2016).

[22] C. Felser, G. H. Fecher, and B. Balke, Spintronics: A Challenge for Materials Science and Solid-State Chemistry, Angew. Chem., Int. Ed. 46, 668 (2007).

[23] S. Chadov, X. Qi, J. Kübler, G. H. Fecher, C. Felser, and S. C. Zhang, Tunable Multifunctional Topological Insulators in Ternary Heusler Compounds, Nat. Mater. 9, 541 (2010).

[24] T. Graf, C. Felser, and S. S. P. Parkin, Simple Rules for the Understanding of Heusler Compounds, Prog. Solid State Chem. 39, 1 (2011).

[25] J. Kübler, G. H. Fecher, and C. Felser, Understanding the Trend in the Curie Temperatures of $\mathrm{Co}_{2}$-Based Heusler Compounds: Ab Initio Calculations, Phys. Rev. B 76, 024414 (2007).

[26] I. Galanakis, P. H. Dederichs, and N. Papanikolaou, Slater-Pauling Behavior and Origin of the Half-Metallicity of the Full-Heusler Alloys, Phys. Rev. B 66, 174429 (2002).

[27] G. H. Fecher, H. C. Kandpal, S. Wurmehl, C. Felser, and G. Schönhense, Slater-Pauling Rule and Curie Temperature of $\mathrm{Co}_{2}$-Based Heusler Compounds, J. Appl. Phys. 99 , 08J106 (2006).

[28] See Supplemental Material at http://link.aps.org/ supplemental/10.1103/PhysRevX.8.041045 for detailed metallographic characterization, crystal orientation, temperature as well as field dependent magnetization and transport characteristics of all the grown single crystals under investigation. We also present a list of potential spin-gapless semiconducting materials, with varying $N_{V}$, that should show a compensated AHC value near $E_{F}$.

[29] R. Yu, W. Zhang, H.-J. Zhang, S.-C. Zhang, X. Dai, and Z. Fang, Quantized Anomalous Hall Effect in Magnetic Topological Insulators, Science 329, 61 (2010).

[30] E. V. Vidal, G. Stryganyuk, H. Schneider, C. Felser, and G. Jakob, Exploring $\mathrm{Co}_{2} \mathrm{MnAl}$ Heusler Compound for 
Anomalous Hall Effect Sensors, Appl. Phys. Lett. 99, 132509 (2011).

[31] G. Chang et al., Room-Temperature Magnetic Topological Weyl Fermion and Nodal Line Semimetal States in HalfMetallic Heusler $\mathrm{Co}_{2} \mathrm{TiX}(X=\mathrm{Si}, G e$, or $\mathrm{Sn}$ ), Sci. Rep. 6 , 38839 (2016).

[32] S. Chadov, S.-C. Wu, C. Felser, and I. Galanakis, Stability of Weyl Points in Magnetic Half-Metallic Heusler Compounds, Phys. Rev. B 96, 024435 (2017).

[33] S. Ouardi, G. H. Fecher, C. Felser, and J. Kubler, Realization of Spin Gapless Semiconductors: The Heusler Compound $\mathrm{Mn}_{2} \mathrm{CoAl}$, Phys. Rev. Lett. 110, 100401 (2013).
[34] T. Jen-Chuan and G. Guang-Yu, High Spin Polarization of the Anomalous Hall Current in Co-Based Heusler Compounds, New J. Phys. 15, 033014 (2013).

[35] Z. Yan, R. Bi, H. Shen, L. Lu, S.-C. Zhang, and Z. Wang, Nodal-Link Semimetals, Phys. Rev. B 96, 041103 (2017).

[36] K. Koepernik and H. Eschrig, Full-Potential Nonorthogonal Local-Orbital Minimum-Basis Band-Structure Scheme, Phys. Rev. B 59, 1743 (1999).

[37] J. P. Perdew, K. Burke, and M. Ernzerhof, Generalized Gradient Approximation Made Simple, Phys. Rev. Lett. 77, 3865 (1996). 